\title{
Prevalence of Overweight and Obesity among School Going Adolescent in Patna
}

\author{
Kumari Mangalam ${ }^{1}$, Chauhan Muneesh ${ }^{2}$, Anand Akshay ${ }^{3}$, Fatima Ambreen ${ }^{1}$, Saho Pramod ${ }^{1}$ \\ ${ }^{1}$ Assistant Professor, Galgotias University, Greater Noida, ${ }^{2}$ Student, Galgotias University, Greater Noida, \\ ${ }^{3}$ Assistant Professor, Manav Rachna International University, Faridabad
}

\begin{abstract}
Introduction: Now a day's Obesity has become pandemic concern globally and the prevention of later stage obesity will require elimination and management of childhood obesity. Childhood obesity and overweight are linked with hypertension, dyslipidemia and decreased metabolism of glucose that ultimately affects our quality of life and physical health and further can lead to increment in the risk of early disease and death.
\end{abstract}

Aims and Objectives: The current investigation was conducted to find out the prevalence of overweight and obesity in school going adolescents.

Material and Method: The present study was a cross-sectional, school-based study conducted in four urban schools of Patna from June, 2019 to, February 2020 with sample size of 660 adolescents (380 boys and 280 girls). The study design was approved by the institutional ethical committee. Three schools accepted our proposal out of 5 schools selected through systematic random sampling. A predesigned and pretested questionnaire was used to collect data on sociodemographic, dietary intake, physical activity and anthropometric measurements.

Result and Discussions: The pervasiveness of overweight and obesity was found to be $18.18 \%(120)$ and $10.9 \%$ (72), respectively in our study. The prevalence of overweight and obesity was $11.3 \%$ and $5.5 \%$, respectively among the males. Males are more affectionate to Non Vegan-compared to females where the number of vegetarian supersedes the animal eater. Calories deficiency is $71.2 \%$ in the whole population on the other hand overeating was seen more in male candidates than females. More than $20 \%$ of the population was found to dine out once or more than once a week .Fast food intake found to be ingested in $44.6 \%$ population once or twice a week on the other hand there is a huge population of $37.8 \%$ who are consuming 3-5 times a week. Mehta et al. revealed the predominance of obesity and overweight as $5.3 \%$ and $15.2 \%$, respectively in his cross-sectional study which was directed in schools of Delhi. Sharma et al demonstrated that $6.4 \%$ school children are obese in Delhi.

Conclusions: The current study suggests that juvenile overweight and obesity is a issue of serious concern in urban community as well as all over. We can prevent this epidemic by doing lifestyle modifications and spreading awareness among people. To achieve this appropriate health education training and awareness program should be imparted to guardians, school authority and children.

Keywords: Obesity, Adolescents,overweight, WHO, Dietary Habits, physical Activity level.

\section{Corresponding Author:}

\section{Akshay Anand}

Assistant Professor (Manav Rachna International University, Faridabad) Tuglakabad Extension Delhi e-mail: akshayaanad.anand25@gmail.com

\section{Introduction}

Presently, Obesity has become a serious and genuine unavoidable concern in all generation groups. Obesity is characterised as a condition wherein there is abnormal or uncurbed accumulation of fat in adipose tissue occurs, to the degree that health may be compromised ${ }^{1}$. This 
worldwide pandemic of excessive weight and obesity -collectively termed as "globesity" - is quickly turning into a significant public health concern in all over the globe.

Obesity has outreached epidemic extent globally, and is a remarkable patron to the chronic ailment and disability having more than 1 billion adults overweight and around 300 million clinically obese ${ }^{1}$. progressively it is perceived as a critical issues in both developing and countries experiencing financial transition ${ }^{2}$.

These issues are not only limited to adults but also being accounted among the children and adolescents of developed ${ }^{3,4}$ as well as developing nations of the world ${ }^{5}$. This new drifting concern lead to various consequences in both adolescence ${ }^{6}$ and adult life ${ }^{7}$ especially increased rate of coronary artery diseases, hypertension, diabetes, dyslipidaemia $^{8}$, obstructive sleep apnoea ${ }^{9}$, esophageal reflux \& gastric emptying disturbances, osteoarthritis $\&$ flat feet ${ }^{10}$, psychological dysfunction, self esteem \& social isolation6, and overall increase in morbidity and mortality in later life ${ }^{11}$.

Epidemiological studies have reported that about a third of preschool children and a half of schoolage children become obese adults ${ }^{12}$. The effective prevention of adult obesity will require the prevention and management of childhood obesity ${ }^{1}$. India has also witnessed a gradual and continuous rise in trends of childhood and adolescent overweight and obesity ${ }^{13}$. Taking this into consideration of these realities, the present study was conducted to the prevalence of Overweight and Obesity in Adolescent School Children of "Patna", capital of Bihar.

\section{Material and Method}

The current study was a cross-sectional, schoolbased study conducted in four urban schools of Patna from June, 2019 to, February 2020 with sample size of 660 adolescents ( 380 boys and 280 girls) with the aims and objectives to determine the prevalence of overweight and obesity in adolescent school children. The study design was approved by the institutional ethical committee. In this study Three schools accepted our proposal out of 5 schools selected through systematic random sampling. Informed consent were taken from the parents prior to the study. Apparently Adolescents school children of Vth to Xth standard, in the range of 8-15 years of age were included in the study. Following Children having Chronic illness, Severe malnutrition, any type of Endocrinal problems, Physical \& Mental disorders and Children with apparent obesity induced or associated with any syndrome were excluded from the study.

The data was collected by using the pre-structured questionnaire to assess total food intake, Type of diet, dietary habits, anthropometric measurement and physical activity.

Information on socio-demographic particulars, dietary habits and usual physical activity, time spent in watching TV and with computer and in other sedentary activities during the past one month, was assessed. Physical activity level (PAL) of the Children were assessed by using Global Physical Activity Questionnaire (GPAQ). Anthropometric measurements of weight $(\mathrm{kg})$ and standing height $(\mathrm{cm})$, waist circumference $(\mathrm{cm})$, were taken by using standard equipment and procedures. Utilizing the weight and height, Body mass index (BMI) was calculated in $\mathrm{Kg} / \mathrm{m} 2$, for each subject.

For overweight and obesity - IOTF classification was considered for calculation of overweight and obese subjects values between 85 th -95 th percentile were defined under overweight. values above 95 th percentile were considered as obese subjects.

\section{Result and Discussion}

Ms Excel was used for Data entry. Statistical Analysis was done using SPSS version 22.0 software for Windows (SPSS Inc., Chicago, IL, USA) and P $<0.05$ was considered as statistically significant. Descriptive statistics were calculated in the form of percentages, mean and standard deviation for the demographic data. A total of 660 adolescent school children were recruited. Out of 660 adolescents interviewed, 53.03 $(\mathrm{n}=350)$ were males and $46.9 \%(n=310)$ were females. The pervasiveness of overweight and obesity was found to be $18.18 \%(120)$ and $10.9 \%(72)$, respectively in our study. The prevalence of overweight and obesity was $11.3 \%$ and $5.5 \%$, respectively among the males. 
Table 1. Mean \pm S.D. Weight, Height, BMI and WC of Females

\begin{tabular}{|c|c|c|c|c|c|}
\hline Age Group & $\mathbf{n}$ & Weight $\mathbf{( k g )}$ & Height $(\mathbf{c m})$ & BMI $\left(\mathbf{k g} / \mathbf{m}^{2}\right)$ & WC $(\mathbf{c m})$ \\
\hline $10-11$ & 49 & $32.10 \pm 6.53$ & $146.78 \pm 9.46$ & $14.90 \pm 2.61$ & $62.36 \pm 7.48$ \\
\hline$>11-12$ & 51 & $37.75 \pm 9.57$ & $152.62 \pm 9.60$ & $16.17 \pm 3.56$ & $64.36 \pm 12.86$ \\
\hline$>12-13$ & 71 & $40.81 \pm 8.96$ & $154.96 \pm 7.49$ & $16.98 \pm 3.57$ & $67.76 \pm 9.87$ \\
\hline$>13-14$ & 68 & $44.81 \pm 11.16$ & $157.14 \pm 8.14$ & $18.11 \pm 4.10$ & $69.87 \pm 9.05$ \\
\hline$>14-15$ & 50 & $48.34 \pm 9.27$ & $160.32 \pm 8.09$ & $18.83 \pm 3.40$ & $72.65 \pm 8.95$ \\
\hline$>15-16$ & 21 & $51.65 \pm 11.42$ & $162.91 \pm 6.27$ & $19.42 \pm 3.96$ & $75.30 \pm 12.35$ \\
\hline Total & $\mathbf{3 1 0}$ & $\mathbf{4 1 . 6 7} \pm \mathbf{1 0 . 9 8}$ & $\mathbf{1 5 5 . 1 0} \pm \mathbf{9 . 4 7}$ & $\mathbf{1 7 . 2 1} \pm \mathbf{3 . 7 9}$ & $\mathbf{6 8 . 0 4} \pm \mathbf{1 0 . 5 6}$ \\
\hline
\end{tabular}

Table 2. MEAN \pm S.D. Weight, Height, BMI and WC of Males

\begin{tabular}{|c|c|c|c|c|c|}
\hline Age Group & $\mathbf{N}$ & Weight (kg) & Height (cm) & BMI $\left(\mathrm{kg} / \mathrm{m}^{2}\right)$ & WC $(\mathrm{cm})$ \\
\hline \multirow{2}{*}{$10-\mathrm{Nov}$} & \multirow{2}{*}{40} & \multirow{2}{*}{$32.90 \pm 11.46$} & 144.35 & 15.5 & 63.15 \\
\hline & & & \pm 9.54 & \pm 4.04 & \pm 11.52 \\
\hline \multirow{2}{*}{$>11-12$} & \multirow{2}{*}{50} & 35.18 & 147.14 & 15.79 & 63.78 \\
\hline & & \pm 7.01 & \pm 16.11 & \pm 2.31 & \pm 6.90 \\
\hline \multirow{2}{*}{$>12-13$} & \multirow{2}{*}{67} & 41.9 & 152.05 & 17.96 & 70.5 \\
\hline & & \pm 10.44 & \pm 9.64 & \pm 3.63 & \pm 10.32 \\
\hline \multirow{2}{*}{$>13-14$} & \multirow{2}{*}{83} & 45.88 & 159.33 & 17.94 & 70.17 \\
\hline & & \pm 11.84 & \pm 10.28 & \pm 3.53 & \pm 10.04 \\
\hline \multirow{2}{*}{$>14-15$} & \multirow{2}{*}{80} & 53.14 & 164.27 & 19.53 & 74.29 \\
\hline & & \pm 13.35 & \pm 6.68 & \pm 3.98 & \pm 14.15 \\
\hline \multirow{2}{*}{$>15-16$} & \multirow{2}{*}{30} & 54.16 & 164.88 & 19.77 & 75.68 \\
\hline & & \pm 14.50 & \pm 6.68 & \pm 4.46 & \pm 13.87 \\
\hline \multirow{2}{*}{ Total } & \multirow{2}{*}{350} & 44.62 & 156.23 & 17.91 & 70.03 \\
\hline & & \pm 13.72 & \pm 12.77 & \pm 3.94 & \pm 12.05 \\
\hline
\end{tabular}

Table 3. Distribution of Study Population According to Age Group, Sex and Type of School

\begin{tabular}{|c|c|c|c|c|c|c|}
\hline \multirow{2}{*}{$\frac{\text { Background Characteristic }}{\text { Age Group (Years) }}$} & \multicolumn{2}{|c|}{ Male } & \multicolumn{2}{|c|}{ Female } & \multicolumn{2}{|c|}{ Total } \\
\hline & $\mathrm{n}$ & $\%$ & $\mathrm{n}$ & $\%$ & $\mathrm{n}$ & $\%$ \\
\hline $8-9 \mathrm{Yr}$ & 40 & 11.42857143 & 44 & 14.19354839 & 84 & 12.72727273 \\
\hline$>9-10$ & 50 & 14.28571429 & 46 & 14.83870968 & 96 & 14.54545455 \\
\hline$>10-11$ & 73 & 20.85714286 & 66 & 21.29032258 & 139 & 21.06060606 \\
\hline$>11-12$ & 70 & 20 & 63 & 20.32258065 & 133 & 20.15151515 \\
\hline$>12-13$ & 70 & 20 & 45 & 14.51612903 & 115 & 17.42424242 \\
\hline$>13-14$ & 20 & 5.714285714 & 16 & 5.161290323 & 36 & 5.454545455 \\
\hline $14-15$ & 27 & 7.714285714 & 30 & 9.677419355 & 57 & 8.636363636 \\
\hline Total & 350 & 53 & 310 & 47 & 660 & 100 \\
\hline
\end{tabular}

Among the females, we found the prevalence of overweight was $7.2 \%$ and that of obesity was $5.4 \%$. The prevalence of overweight and obesity was found to high among males.
Among the females, we found the prevalence of overweight was $7.2 \%$ and that of obesity was $5.4 \%$. The prevalence was found to high among the males. Males are more affectionate to Non Vegan-compared to 
females where the number of vegetarian supersedes the animal eater. Calories deficiency is $71.2 \%$ in the whole population on the other hand overeating was seen more in male candidates than females. More than $20 \%$ of the population was found to dine out once or more than once a week .Fast food intake found to be ingested in $44.6 \%$ population once or twice a week on the other hand there is a huge population of $37.8 \%$ who are consuming 3-5 times a week. Only $36.3 \%$ candidates were consuming fruits 5 times a week. Around $43 \%$ of Population in our study were performing moderate/vigorous activities remaining were performing the same activity level and they are $56 \%$ of the population. $50 \%$ of Population were performing moderate PA, while $28.4 \%$ were stuck on Low PA, Only 20.7\% were performing High PA.13.3\% of candidates uses mobile phone more than 2 hours a day while $42.7 \%$ of population still uses 1 hours every day. A major section of population doesn't uses computer i.e. $50.4 \%$ while $12.5 \%$ uses more than a hour.

Total sedentary time was $56.3 \%$ in $6-10$ hours user. While $35.7 \%$ is the percentage of least user.

\section{Discussion}

India, considered as an ethnic group are mainly at greater risk for insulin resistance and central obesity, Prompting to diabetes, Heart Disease and other "life style" disorders. Over nutrition and sedentary lifestyle predisposes to various metabolic syndrome and type 2 DM. various investigations have demonstrated that these problems begins in childhood and manifest due to interactions and accumulation of multiple risk factors, throughout the life span. Mehta et al. revealed the predominance of obesity and overweight as 5.3\% and $15.2 \%$, respectively in his cross-sectional study which was directed in schools of Delhi ${ }^{14}$.Sharma et al demonstrated that $6.4 \%$ school children are obese in Delhi ${ }^{15}$.

In our study the event of overweight and obesity was found to be $18.18 \%$ and $10.9 \%$, respectively. Perhaps this is on the ground that in Patna city there is accessibility of wide range of fast/junk food corners, modern electronic gadgets, games and transport facilities which are contributing for the increased prevalence of overweight and obesity in adolescents.

Similar finding were accounted by Goyal et al. with $12 \%$ of overweight and obesity among adolescents (aged 12-18 years) in addition to this It was also reported that the prevalence was higher in children who belongs to high socioeconomic class as compared to those who belongs to the lower socioeconomic class ${ }^{16}$.

The predominance of overweight and obesity among the school-going adolescents of Patna was seen as high . Raj et al indicated a gradual increment pattern transition in health concerning with overweight and obesity ${ }^{13}$. Among all sedentary actions, TV viewing has received unique attention, and in this study, it was seen as to be a significant independent factor influencing overweight and obesity.

Overweight is characterized as a BMI between the 85th and 95th percentile for age and sex, and obesity-as a BMI greater than the 95 th percentile. BMI is utilized as a particular pointer of abundance adiposity among children.

The prevalence rates of adolescents overweight and obesity in our region (Patna) were higher as compared to previously reported data from other areas in Bihar.

\section{Conclusion and Recommendations}

We Concluded that whether the city is enormous or small this is a genuine concern to be dealt with. It is very essential to take preventive measures such as awareness programs school based and community based to avoid this crucial issue of overweight and obesity. To sum up, our study also conclude that restricted physical movement, undisciplined and detrimental food tendency and increment in stationary and inactive way of life are turning out to be major contributing element for overweight and obesity. The current study suggests that juvenile overweight and obesity is a issue of serious concern in urban community as well as all over. We can prevent this epidemic by doing lifestyle modifications and spreading awareness among people. To achieve this appropriate health education training and awareness program should be imparted to guardians, school authority and children.

Limitations: For more vital and precise information larger sample size could have taken. however due to lockdown same could not be achieved. Information about the dietary habits, intake and others must be taken in front of guardian for accurate information.

Acknowledgment: We would like to thank all the participants of the study for their cooperation. Financial support and sponsorship Nil. Conflicts of interest There are no conflicts of interest.

Ethical Clearance: Institutional ethical committee 


\section{References}

1. World Health Organization. Obesity: preventing and managing the global epidemic. World Health Organization; 2000.

2. Popkin BM. The nutrition transition and obesity in the developing world. The Journal of nutrition. 2001 Mar;131(3):871S-3S.

3. Popkin BM, Gordon-Larsen P. The nutrition transition: worldwide obesity dynamics and their determinants. International journal of obesity. 2004 Nov;28(3):S2-9.

4. Ogden CL, Carroll MD, Curtin LR, McDowell MA, Tabak CJ, Flegal KM. Prevalence of overweight and obesity in the United States, 1999-2004. Jama. 2006 Apr 5;295(13):1549-55.

5. Wang Y, Lobstein TI. Worldwide trends in childhood overweight and obesity. International journal of pediatric obesity. 2006 Jan 1;1(1):11-25.

6. Dietz WH. Health consequences of obesity in youth: childhood predictors of adult disease. Pediatrics. 1998 Mar 1;101(Supplement 2):518-25.

7. Must A, Jacques PF, Dallal GE, Bajema CJ, Dietz WH. Long-term morbidity and mortality of overweight adolescents: a follow-up of the Harvard Growth Study of 1922 to 1935. New England journal of medicine. 1992 Nov 5;327(19):13505. Lauer RM, Clarke WR, Mahoney LT, Witt J. Childhood predictors for high adult blood pressure: The Muscatine Study. Pediatric Clin North Am 1993; 40: 23-40.

8. Roland C, Baur LA, Donaghue KC, Waters KA. Metabolic correlates with obstructive sleep apnea in obese subjects. The Journal of pediatrics. 2002 Jun 1;140(6):654-9.
9. Dowling AM, Steele JR, Baur LA. Does obesity influence foot structure and plantar pressure patterns in prepubescent children?. International journal of obesity. 2001 Jun;25(6):845-52.

10. Burton BT, Foster WR. Health implications of obesity: an NIH Consensus Development Conference. Journal of the American Dietetic Association. 1985 Sep;85(9):1117-21.

11. Serdula MK, Ivery D, Coates RJ, Freedman DS, Williamson DF, Byers T. Do obese children become obese adults? A review of the literature. Preventive medicine. 1993 Mar 1;22(2):167-77.

12. Raj M, Sundaram KR, Paul M, Deepa AS, Kumar RK. Obesity in Indian children: time trends and relationship with hypertension. National Medical Journal of India. 2007 Nov 1;20(6):288.

13. Mehta M, Bhasin SK, Agrawal K, Dwivedi S. Obesity amongst affluent adolescent girls. Indian JPediatr. 2007;74:619-22.

14. Sharma A, Sharma K, Mathur KP. Growth pattern and prevalence of obesity in affluent schoolchildren of Delhi. Public Health Nutr. 2007;10:485-91.

15. Prasad RV, Bazroy J, Singh Z. Prevalence of overweight and obesity among adolescent students in Pondicherry, South India. International Journal of Nutrition, Pharmacology, Neurological Diseases. 2016 Apr 1;6(2):72.

16. Goyal JP, Kumar N, Parmar I, Shah VB, Patel B. Determinants of overweight and obesity in affluent adolescent in Surat City, South Gujarat region, India. Indian J Community Med. 2011;36:296-300 